\title{
BLOCKCHAIN: UNA REVOLUCIÓN DE VIEJA DATA
}

\section{BLOCKCHAIN: UNA REVOLUCIÓN DE VIEJA DATA ${ }^{12}$}

\author{
BLOCKCHAIN: AN OLD REVOLUTION
}

Santiago Grigera del Campillo(*)

\begin{abstract}
RESUMEN: La revolución de la tecnología blockchain fue producto del desarrollo de técnicas matemáticas que se fueron complementando con otras tecnologías que ha llevado a organizaciones públicas y privadas a reconducir y adaptar sus infraestructuras y modelo de negocios. El derecho argentino ha evolucionado lentamente, pero de a poco intenta ponerse al día.
\end{abstract}

PALABRAS CLAVES: Blockchain- Criptografía- Firma digital- Firma Electrónica- Seguridad- Equivalencia funcional- Autoridad de sello de tiempo- Servicios de Confianza

ABSTRACT: The blockchain technology revolution was the product of the development of mathematical techniques that were complemented with other technologies that has led public and private organizations to redirect and adapt their infrastructures and business model. Argentine law has evolved slowly, but little by little it tries to catch up.

KEY WORDS: Blockchain- Cryptography- Digital signature- Electronic signature- Security- Functional equivalence- Time stamp authority- Third party services

SUMARIO: I. Introducción. II. Firma electrónica y digital. Marco Normativo relevante y actual. III. Servicios de confianza. IV. Firma electrónica y Blockchain. V. Reflexiones finales.

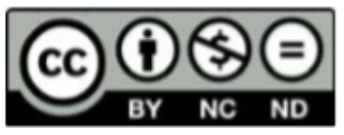

Artículo publicado bajo Licencia Creative Commons Atribución-No Comercial-Sin Derivar. (C) Universidad Católica de Córdoba DOI http://doi.org/10.22529/rbia.2021(2)01

\footnotetext{
${ }^{1}$ Artículo recibido el 9 de marzo de 2021 y aprobado para su publicación el 30 de junio de 2021

${ }^{2}$ El presente es trabajo presentado se realizó en el marco de la DIPLOMATURA EN TECNOLOGÍA BLOCKCHAIN APLICADA A LOS NEGOCIOS Y LAS RELACIONES JURIDICAS, en el año 2020.

(*) Abogado (UNC). Especialista en Derecho Informático (Universidad de Buenos Aires). Profesor en la Diplomatura en Tecnología Blockchain aplicada a los negocios y las relaciones (UCCOR), Profesor en Diplomatura de Derecho Digital: Herramientas teóricas prácticas (Acción Jurídica). Prosecretario de la Sala de Derecho y Tecnología del Colegio de Abogados de Córdoba. Maestrando en Derecho de la Ciberseguridad y entorno digital, Universidad de León, España.
} 


\section{BLOCKCHAIN: UNA REVOLUCIÓN DE VIEJA DATA}

\section{INTRODUCCIÓN}

Blockchain es una palabra intimidante, novedosa y con valor intrínseco que aumenta exponencialmente. Las industrias consolidadas intentan contrarreloj no quedarse afuera (Síndrome FOMO- "Fear of Missing Out") y sumarse al desafío de transformar sus negocios concebidos de manera analógica, porque hoy compiten con nuevos proyectos y empresas nacidas a partir de las virtudes de esta tecnología.

Resulta al menos extraño, que estemos transitando por lo que hoy en día muchos llaman la nueva revolución de internet cuando la realidad demuestra que bitcoin cumplirá 12 años en el 2020. Asimismo, la idea de "cash digital”, que se destaca por ser "descentralizado y anónimo" se remonta al año 1982, en el que el Criptógrafo David Lee Chaum publicaba su white paper "Blind signatures for untraceable payments" ${ }^{\prime 3}$ donde proponía una nueva idea de criptografía en transacciones en pos de garantizar la privacidad de las personas. El término "Smart Contracts" fue acuñado por Nick Szabo en 1997 en un artículo llamado "Formalizing and Securing Relationships on Public Networks"4. Más aún, no se puede concebir a blockchain sin criptografía, concebida vulgarmente como el arte de ocultar información para evitar que sea accedida por receptores no autorizados. Este método de orígenes inciertos, encuentran antecedentes en la antigua Grecia, pasando por el Imperio Romano y ha tenido revelaciones en las Guerras Mundiales y continúa vigente de manera perfeccionada a la fecha.

Con todo esto quiero decir que la "nueva" tecnología blockchain está constituida a base de componentes viejos que lo nutren de seguridad y confianza.

3 Chaum David Lee (1982). "Blind signatures for untraceable payments". Recuperado de http://www.hit.bme.hu/ buttyan/courses/BMEVIHIM219/2009/Chaum.BlindSigForPayment.1982.PDF . Consultado el 15/04/2020.

${ }^{4}$ Szabo, N. (1997). Formalizing and Securing Relationships on Public Networks. First Monday, 2(9). Recuperado de https://journals.uic.edu/ojs/index.php/fm/article/view/548/469. Consultado el 15/04/2020. 


\section{BLOCKCHAIN: UNA REVOLUCIÓN DE VIEJA DATA}

Uno de los aspectos desde donde podemos abordar a la cadena de bloques es analizando uno de esos elementos que le proporciona seguridad. En el presente artículo, me referiré brevemente a la firma electrónica y su relación con la tecnología blockchain brindando una noción a los aspectos legales que la circundan.

\section{FIRMA ELECTRÓNICA Y DIGITAL. MARCO NORMATIVO ARGENTINO RELEVANTE Y ACTUAL.}

En Argentina la ley de firma digital N² 25.506 desarrolla especialmente este concepto y los de firma electrónica, documentos y certificados digitales, autoridades de aplicación, infraestructura, competencias, facultades, entre muchas otros. Un dato no menor es que la ley fue sancionada el 14/11/2001 y promulgada en diciembre del mismo año. En el 2020 resulta extraño que podamos relacionar algo tan novedoso como blockchain con una ley de más de 19 años. Vale la pena mencionar, que además ha sido modificada y derogada parcialmente en varias oportunidades, también sus decretos reglamentarios.

La firma digital, ha sido conceptualizada en el artículo 2 de la siguiente manera: “...resultado de aplicar a un documento digital un procedimiento matemático que requiere información de exclusivo conocimiento del firmante, encontrándose ésta bajo su absoluto control. La firma digital debe ser susceptible de verificación por terceras partes..."

Tal vez la denominación "firma" nos resulte confusa porque nos lleva a asociarla necesariamente a la firma de puño y letra, cuando en realidad es un complemento que se le agrega a un documento digital como consecuencia de un procedimiento matemático que identifica a quien lo ha realizado y proporciona una especie de escudo para evitar que dicho documento sea modificado. Además, interviene toda una infraestructura con autoridades y jerarquías que fortalecen los actos en los que se utiliza. Por otro lado, la firma electrónica ha sido conceptualizada en el art. 5 como un conjunto de datos electrónicos integrados o asociados de manera lógica a otros, utilizado por el signatario como su medio de identificación y que carezca 


\section{BLOCKCHAIN: UNA REVOLUCIÓN DE VIEJA DATA}

de los requisitos para ser considerado firma digital. Así, este tipo emerge como una alternativa más informal y sin intervención de estructuras validantes lo que implica que ante un desconocimiento de la misma, el que la invoca tiene que acreditar su autoría e integridad. La firma electrónica tiene aplicaciones desde un email hasta un botón de un sitio web donde se aceptan las condiciones para acceder a un servicio o al comprar un producto online.

Es adecuado reparar en lo que se ha denominado como principio de "equivalencia funcional". A groso modo, implica que si se cumple el mismo cometido corresponde dotarlo del mismo efecto jurídico independientemente del soporte. Como antecedente internacional encuentra orígenes en las leyes modelo CNUDMI (UNCITRAL por sus siglas en inglés) de comercio electrónico y firmas electrónicas. El artículo 3 de la ley de firma digital, sostiene que cuando la ley requiera una firma manuscrita, esa exigencia también queda satisfecha por una firma digital. Autores como Daniel R. Altmark y Eduardo Molina Quiroga ${ }^{5}$, cuestionan la redacción de este artículo indicando que a su criterio, la norma debe interpretarse de la misma manera respecto de la firma electrónica.

El Código Civil y Comercial, ha previsto la libertad de formas a los fines de exteriorización de voluntad siempre que no exista una forma impuesta por ley (art.284). Esto resulta lógico. En la época antigua se aceptaban como declaraciones de voluntad las efectuadas sobre piedra o papiro, no existe óbice para incluir también la manifestación verbal. Todo será una cuestión de prueba si fuere menester. El CCCN introduce que la expresión escrita puede hacerse constar en cualquier soporte. Al respecto, el comentario al art $286^{6}$ es muy ilustrativo: "Este artículo introduce el concepto de soporte, y por ende el de la grafía, con un criterio muy amplio: todo aquel elemento que permita representar el contenido de modo que los sujetos a los

\footnotetext{
${ }^{5}$ Altmark Daniel R. \& Molina Quiroga E. (2012). Capítulo III. Documentos electrónicos y Firma digital. En Tratado de Derecho Informático. Tomo I. (pp. 593-594). Buenos Aires, Argentina: La Ley.

${ }^{6}$ D’alessio Carlos Marcelo (2015). Código Civil y Comercial de la Nación comentado. Tomo II. (pp. 114-115). Buenos Aires, Argentina: Rubinzal Culzoni Editores.
} 


\section{BLOCKCHAIN: UNA REVOLUCIÓN DE VIEJA DATA}

que va dirigido puedan enterarse del mismo, aun cuando para ello deban emplear medios técnicos. De este modo se incluye en la categoría de instrumentos a los llamados documentos electrónicos: aquellos en los cuales la manifestación de voluntad es emitida a través de un sistema de computación, por lo que carecen de materialidad y su contenido, al que sólo puede accederse a través de medios tecnológicos informáticos, está habitualmente resguardado por procedimientos de seguridad.

El articulado del Código continúa referenciando los instrumentos privados y los particulares no firmados y en el art. 287 alude sobre estos últimos de una manera muy amplia con independencia del medio empleado. De todo este derrotero terminamos por derivar en el art. 288 donde se le reconoce a la firma digital los mismos efectos que la firma ológrafa. Y en este punto, en el Código Civil y Comercial comentado, dirigido por el Dr. Lorenzetti, uno de los autores del capítulo donde desarrolla el artículo que se analiza, deja una ventana abierta para el futuro expresando lo siguiente: “... la terminología utilizada en la norma deberá interpretarse inclusiva de cualquier procedimiento que se desarrolle en el futuro que asegure autoría e integridad del documento aun cuando sus características técnicas sean diferentes a la firma digital conocida en la actualidad"7. Concretamente, establece que mientras un procedimiento a futuro garantice la integridad y autoría el requisito de firma se considerará satisfecho mediante ese procedimiento.

En materia de contratos de consumo, Título III CCCN, encontramos varios supuestos que aluden a este principio de equivalencia funcional, en todo lo que refiera a contratos a distancia, contratación electrónica (arts. 1105-1109). También en materia probatoria, se destaca la libertad de cumplimentarlo por cualquier medio conforme surge de los arts. 1019 y 1020. Asimismo, en cuanto a la fecha cierta el art. 317, permite que dicha circunstancia sea probada por cualquier medio mientras surja que el documento se encontraba firmado. Este artículo es

\footnotetext{
${ }^{7}$ D’alessio Carlos Marcelo (2015). Código Civil y Comercial de la Nación comentado. Tomo II. (pp. 121). Buenos Aires, Argentina: Rubinzal Culzoni Editores.
} 


\section{BLOCKCHAIN: UNA REVOLUCIÓN DE VIEJA DATA}

relevante respecto de las autoridades de sello de tiempo. Por último, no se puede dejar de mencionar el art. 77 del Código Penal Argentino, donde en el título XIII refiere a la significación de conceptos empleados en el código. Al respecto se establecen las siguientes precisiones: El término "documento" comprende toda representación de actos o hechos, con independencia del soporte utilizado para su fijación, almacenamiento, archivo o transmisión. Los términos "firma” y "suscripción" comprenden la firma digital, la creación de una firma digital o firmar digitalmente. Los términos "instrumento privado" y "certificado" comprenden el documento digital firmado digitalmente. Con todo lo enunciado no quería dejar de resaltar la vasta recepción normativa que contempla la eficacia jurídica sobre la utilización de medios electrónicos en estos tiempos donde las expresiones analógicas o en formato papel han quedado postergadas y reducidas a algunos pocos actos solemnes.

Continuando, sin perjuicio que no abordaré todos los cambios de esta ley especial por una cuestión de pertinencia con el tema desarrollado, podemos destacar que en su primer decreto reglamentario $N^{\circ} 2628 / 02$ ya hacía referencia al término “terceros confiables" en su art. 5.

En consonancia con lo mencionado, a través de la decisión administrativa de Jefatura de Gabinete 927/14 determina a los servicios de terceros para la prestación de otros servicios en relación de la firma digital. En el artículo 10, determina a los certificados de aplicaciones, a los sellos de tiempo y de competencia. Estas incorporaciones han sido aclaratorias y a partir de estas novedades, aumentaron la utilización en el sector privado y en Estado a través de los distintos decretos reglamentarios como el 561/16, 1063/16 (donde regularon el sistema de gestión documental electrónica -GDE- la plataforma TAD de trámites a distancia), Decreto $\mathrm{N}^{\circ}$ 892/2017 de creación de la Plataforma de firma digital remota entre otras.

Lo mencionado en el párrafo anterior guarda relación con lo establecido en el artículo 317 CCCN comentado anteriormente, respecto de la manera en que se puede probar la fecha cierta. 


\section{BLOCKCHAIN: UNA REVOLUCIÓN DE VIEJA DATA}

El Parlamento Europeo ha regulado con un poco más en detalle sobre la materia firma electrónica, sello de tiempo, y servicios de confianza en el REGLAMENTO (UE) 910/2014 DEL PARLAMENTO EUROPEO Y DEL CONSEJO relativo a la identificación electrónica y los servicios de confianza para las transacciones electrónicas en el mercado interior. Se exhorta a consultar dicha fuente desde que explica con precisión los temas analizados ${ }^{8}$. Es importante destacar que en Europa el término firma electrónica difiere del asignado a en la ley Nacional 25.506. Para empezar en el reglamento no está previsto el término firma digital. La firma electrónica cualificada Europea es la que se parecería más a lo que localmente conocemos como firma digital.

Como si la ley no hubiera sufrido suficientes alteraciones, posteriormente se sancionó la ley 27.446, con ánimo de desburocratizar el Estado en el año 2018. Produjo varios cambios a la ley 25.506, como la derogación de los arts. $4^{\circ}$ (importante porque derogó exclusiones de aplicación de firma digital sobre supuestos que estaban reservadas para firmas con solemnidades específicas), 18, 28, 35 y 36 y sustituciones del 10, 27, 29, 30 inc B), 34, entre otros cambios como en materia de títulos valores.

Para ir acercándonos a la actualidad, un nuevo decreto reglamentario apareció con importantes cambios. El decreto 182/19 surgió como consecuencia de integrar todos los cambios producidos en los decretos mencionados y para adaptar a las reformas instrumentadas por la ley 27.446. A modo de comentario previo, se menciona que en el art. 2 establecía que la firma digital suplía la certificación de firmas en los documentos digitales. Particularmente, este artículo fue cuestionado y motivo de planteos judiciales por parte de escribanos ${ }^{9}$. Como

\footnotetext{
${ }^{8}$ Diario Oficial de la Unión Europea. REGLAMENTO (UE) No 910/2014 DEL PARLAMENTO EUROPEO Y DEL CONSEJO de 23 de julio de 2014. Recuperado de https://eur-lex.europa.eu/legalcontent/ES/TXT/PDF/?uri=CELEX:32014R0910\&from=IT. Consultado el 16/04/2020.

${ }^{9}$ Causa: Colegio de Escribanos de la Ciudad de Buenos Aires C/ Estado Nacional Jefatura de Gabinete de Ministros S/ incidente de Medida Cautelar. Juzgado Civil y Comercial Federal 5 Poder Judicial de la Nación Expte 4451/2019; y Causa: Consejo Federal del Notariado Argentino c/ Estado Nacional S/ Acción meramente declarativa-Juzgado Civil y Comercial Federal 5 Poder Judicial de la Nación Expte:6411/19.
} 


\section{BLOCKCHAIN: UNA REVOLUCIÓN DE VIEJA DATA}

consecuencia, se modificó ese artículo mediante un nuevo decreto reglamentario Nro. 774/2019 que determinó la equiparación exclusivamente para trámites efectuados ante la Administración Pública Nacional.

\section{SERVICIOS DE CONFIANZA}

A pesar de lo mencionado, el decreto 182/19 establece algunas normas interesantes en sus anexos que resultan promisorias para la implementación de la tecnología blockchain.

Por ello, sin perjuicio de otras incorporaciones la más pertinente al módulo desarrollado la encontramos en el artículo 36 donde determina a los servicios de confianza, y específicamente en el inc. 5 dónde expresa: “....servicios electrónicos prestados por terceros de confianza relativos a: ...5) La operación de cadenas de bloques para la conservación de documentos electrónicos, gestión de contratos inteligentes y otros servicios digitales”.

A pesar que nos enfocaremos en este inciso, corresponde transcribir el artículo 36, que establece lo siguiente:

"ARTÍCULO 36.- Servicios de Confianza. Se entiende por Servicio de Confianza al servicio electrónico prestado por un tercero de confianza relativo a:

1. La conservación de archivos digitales.

2. La custodia de declaraciones de voluntad realizadas en formato electrónico, contratos electrónicos, y toda otra transacción que las partes decidan confiar a un tercero depositario.

3. La notificación fehaciente de documentos electrónicos.

4. El depósito de declaraciones de voluntad realizadas en formato electrónico.

5. La operación de cadenas de bloques para la conservación de documentos electrónicos, gestión de contratos inteligentes y otros servicios digitales.

6. Los servicios de autenticación electrónica.

7. Los servicios de identificación digital.

8. Otras prestaciones que determine el Ente Licenciante." 


\section{BLOCKCHAIN: UNA REVOLUCIÓN DE VIEJA DATA}

Para una inmersión en el término terceros de confianza, me remito al concepto formulado por el Dr. Gastón Bielli que lo define como: “aquellos sistemas informáticos accesibles vía web, ya sean públicos o privados, que mediante la implementación de tecnologías tales como la firma electrónica, el sellado de tiempo (timestamp), conexiones seguras y mecanismos de depósito electrónico -en forma conexa y en atención a determinados estándares de seguridad- hacen las veces de certificadores y depositarios de documentos electrónicos pasibles de atestiguar la ocurrencia de hechos u actos jurídicamente relevantes suscitados en el mundo virtual $y$, consecuentemente, revestirlos del necesario valor probatorio a fin de eventualmente procurar ser introducidos, como prueba instrumental, a un proceso judicial"10.

Como consecuencia de la volatilidad de la información en los medios electrónicos, surgen estas soluciones para revestir de certidumbre al mundo virtual. Los terceros de confianza representan seguridad y es por eso que inspiran confianza. La ley se los reconoce, ya sea, por las cuestiones técnicas que los constituyen, como por el cumplimiento de ciertos estándares reconocidos a nivel internacional. En el artículo citado, se podrá encontrar jurisprudencia Nacional e Internacional donde se han resuelto casos tomando como servicio de confianza al sitio web Archive.org. El sitio funciona como un repositorio de captura de pantallas y registra las publicaciones que se hacen en todos los sitios de internet a lo largo del tiempo.

Volviendo sobre el inciso 5, es la primera manifestación normativa específica donde se reconoce legalmente la funcionalidad de la cadena de bloques como repositorio, gestión de datos y otros servicios. Por alguna razón que se desconoce han dejado el final abierto y salvo se vuelva a reformar la norma, se evidencia una fuente de nuevas oportunidades para todos los sectores. La posibilidad de servicios que pueden prestarse en blockchain ha quedado indeterminada respecto a los distintos prestadores de confianza lo que supone que tanto particulares como organismos públicos y empresas privadas pueden empezar a explotar este terreno. Sin dudas las

\footnotetext{
${ }^{10}$ Gastón Bielli (2019). Publicado en el Diario La Ley 6/06/2019. Cita online: AR/DOC/1629/2019.Recuperado http://www.iadpi.com.ar/index.php/2020/03/10/wayback-machine/. Consultado el 14/04/2020.
} 


\section{BLOCKCHAIN: UNA REVOLUCIÓN DE VIEJA DATA}

bondades de esta tecnología representan seguridad, inmutabilidad, transparencia, trazabilidad de los procesos incorporados, lo que permite un contexto menos propicio para el fraude, manipulación de datos e información, reconocimiento de derechos como la propiedad intelectual, entre otros. En correlación con lo aquí mencionado, el art.37 establece: “ARTÍCULO 37.- Prestadores de Servicios de Confianza. Podrán brindar servicios de confianza las personas humanas, jurídicas, consorcios, entes públicos, entes públicos no estatales, de acuerdo a los procedimientos, estándares y condiciones que determine la SECRETARÍA DE MODERNIZACIÓN ADMINISTRATIVA de la SECRETARÍA DE GOBIERNO DE MODERNIZACIÓN de la JEFATURA DE GABINETE DE MINISTROS.” De esta manera se aprecia como se ha ampliado el panorama de sujetos legitimados para prestar un servicio de confianza aún incluyendo tecnología tan innovadora como blockchain.

\section{FIRMA ELECTRÓNICA Y BLOCKCHAIN}

Para ir cerrando, de lo desarrollado en el presente artículo podemos concluir que definitivamente en Blockchain no se utiliza firma digital de acuerdo al Derecho Argentino. Se utiliza firma electrónica, tomando como base criptografía de infraestructura de clave pública.

A modo ilustrativo destacando la claridad de la explicación transcribo un párrafo obtenido de un artículo del Blog de MIT technology review donde explica el uso de criptografía en la blockchain: "La criptografía de clave pública se basa en parejas de claves, una pública y otra privada, que son utilizadas para autenticar usuarios y verificar sus transacciones cifradas. Los usuarios de Bitcoin están representados dentro de la cadena de bloques por conjuntos de caracteres llamados direcciones, que son derivados de sus claves públicas. Las aplicaciones 


\section{BLOCKCHAIN: UNA REVOLUCIÓN DE VIEJA DATA}

financieras que se usan para guardar e intercambiar monedas digitales son esencialmente sistemas de gestión de esas claves privadas $(. . .)^{11}$.

\section{REFLEXIÓN FINAL}

Con todo, la primera conclusión que decanta con claridad es que en blockchain se utiliza, según la normativa Argentina, firma electrónica y no una firma digital. Por otro lado, nos encontramos en una época inmejorable para adoptar las virtudes de la tecnología blockchain en todos los sectores. Como vimos, esta revolución ha sido gestada producto de la complementación de recursos antiguos que hoy representan una solución para muchos sectores desde que brindan transparencia, celeridad, seguridad jurídica, evitando cambios arbitrarios o manipulaciones de datos. Tenemos las herramientas disponibles para optimizar nuestro trabajo, y aunque sin dudas quedan cuestiones aún por resolver es importante involucrarse y tratar de ser parte de esta revolución que es una realidad.

\footnotetext{
${ }^{11}$ Mike Orcutt (2017). “'Blockchain' podría derrocar a las contraseñas y devolver la identidad digital sus usuarios”. Traducido por Teresa Woods. Recuperado de https://www.technologyreview.es/s/9821/blockchain-podriaderrocar-las-contrasenas-y-devolver-la-identidad-digital-sus-usuarios. Consultado el 20/04/2020.
} 